Communications in Physics, Vol. 29, No. 1 (2019), pp. 71-76

DOI:10.15625/0868-3166/29/1/13503

\title{
WETTING CONTROL IN THE LAYERED POLYMER-SILVER THIN FILM VIA FEMTOSECOND LASER MICROSTRUCTURING
}

\author{
SOFIA FILIPPOVNA UMANSKAYA ${ }^{1,2, \dagger}$, PAVEL ALEKSANDROVICH DANILOV ${ }^{1}$, \\ SERGEY IVANOVICH KUDRYASHOV ${ }^{1,2,3}$, ANDREY ANDREEVICH RUDENKO ${ }^{2}$ \\ AND ANDREY ALEKSEEVICH IONIN ${ }^{1}$ \\ ${ }^{1}$ Lebedev Physical Institute, Leninskiy prospect 53, 119991 Moscow, Russia \\ ${ }^{2}$ National Research Nuclear University MEPhI (Moscow Engineering Physics Institute), \\ Kashirskoe shosse 31, 115409 Moscow, Russia \\ ${ }^{3}$ ITMO University, Kronverksky prospect 49, 197101 Saint-Petersburg, Russia \\ ${ }^{\dagger}$ E-mail: umanskaya@lebedev.ru
}

Received 25 December 2018

Accepted for publication 21 February 2019

Published 15 March 2019

\begin{abstract}
Water wetting of a structured multi-layered thin film consisting of bottom silver and top polymer layers microstructured by femtosecond laser pulses was studied. The micro-structure of periodic trenches was ablatively produced on the top polymer layer of this film using $515 \mathrm{~nm}$, $220 f$ s pulses generated by the second harmonic of an ytterbium-doped fiber laser at the pulse repetition rate of $20 \mathrm{kHz}$. The topography of the micro-structured film was observed by means of a JEOL $7001 \mathrm{~F}$ scanning electron microscope and its water wetting angles were measured by sideview microscopic imaging. The wetting angle on the microstructured surface was $144^{\circ}$, comparing to $61^{\circ}$ at the raw unstructured surface of the film.
\end{abstract}

Keywords: thin films, metal, polymer, femtosecond laser, femtosecond microstructuring, wettability, hydrophobicity.

Classification numbers: 06.60.Jn, 78.47.J-, 81.16.-c.

(C)2019 Vietnam Academy of Science and Technology 


\section{INTRODUCTION}

Wettability properties of surfaces and in particular their superhydrophobic properties have attracted significant attention because of their significant potential for practical applications such as self-cleaning, anti-corrosion and anti-bacterial. The wettability of a surface depends on its chemical structure as well as its topology and can be characterized by a contact angle. A hydrophilic surface has a contact angle below $90^{\circ}$. A surface with a contact angle higher than $90^{\circ}$ is known as a hydrophobic surface, while for superhydrophobic surface contact angle should be higher than $150^{\circ}$. Generally, surface wettability can be tuned toward superhydrophobic properties by fabrication of micrometer- and nanometer-scale surface structures. Laser micro- and nanostructuring of surfaces provide such coatings [1-4], enabling to scale up for industry this highly reproducible, simple and fast process.

Polymers have many applications in industry due to their low cost, low weight and flexibility, appearing as good materials for laser micromachining and fabricating photonic devices [5]. Their functionally can be added up by thin films of noble metals such as silver and gold [6], exhibiting important modalities in nanooptics and biophotonics due to their optical and plasmonic properties, e.g., arrays of holes produced by femtosecond lasers on silver films have applications in biosensorics $[7,8]$. In many cases, pre-concentration of analyte molecules is required prior plasmonic sensing, which could be achieved via controlled (directional) wettability [9-11].

In this paper, we study the effect of femtosecond-laser micro-structuring of a layered silverpolymer film on its wetting angle for its further potential applications in chemo- and biosensing.

\section{EXPERIMENT}

The target was a thin two-layered film of bottom silver and top polymer layers deposited onto a silica glass slide by magnetron sputtering. The thickness of each layer is $\sim 1$ micron. Laser structuring of the target surface was performed at the workstation for laser micro- and nanostructuring (Fig. 1). The target was installed on a three-dimensional motorized platform with stepper motors with a minimum step of $150 \mathrm{~nm}$, which provides movement of the sample from laser pulse to laser pulse. The laser treatment of the target surface was carried out by pulses of the second harmonic of a Satsuma (Amplitude System) femtosecond laser with an active medium doped with ytterbium ions. Laser parameters were as follows: second-harmonic wavelength - 515 $\mathrm{nm}$, pulse duration of $200 \mathrm{fs}$, maximum pulse energy in $\mathrm{TEM}_{00}$ mode $-4 \mu \mathrm{J}$, pulse repetition rate changeable in the range of $0-2 \mathrm{MHz}$. The laser pulses passed through an energy attenuator, which allows variation of their energy, then the laser pulses were focused on the sample in air through a microscope objective with an aperture of $\mathrm{NA}=0.25$ into a circular spot with a $1 / \mathrm{e}$ radius $R_{1 / e} \approx 2.2 \mu \mathrm{m}$. The produced microstructures were observed by a scanning electron microscope (SEM) JEOL 7001F.

Wetting angles of the microstructured film were measured using side-view imaging of deionized water droplets of diameter about $1.5 \mathrm{~mm}$ on its micro-structured surface by a microscope "Expert" (Prima Expert). The setup for the wetting angle measurements is shown in Fig.2. The sample was placed on a stage that can move along the $\mathrm{z}$ axis in front of the objective of the microscope, the image from which is displayed on the PC.

Workstation for nano- and micro-structuring: PC - personal computer for controlling the laser and 3D platform; BS - beam splitter; CCD - camera for surface visualization 


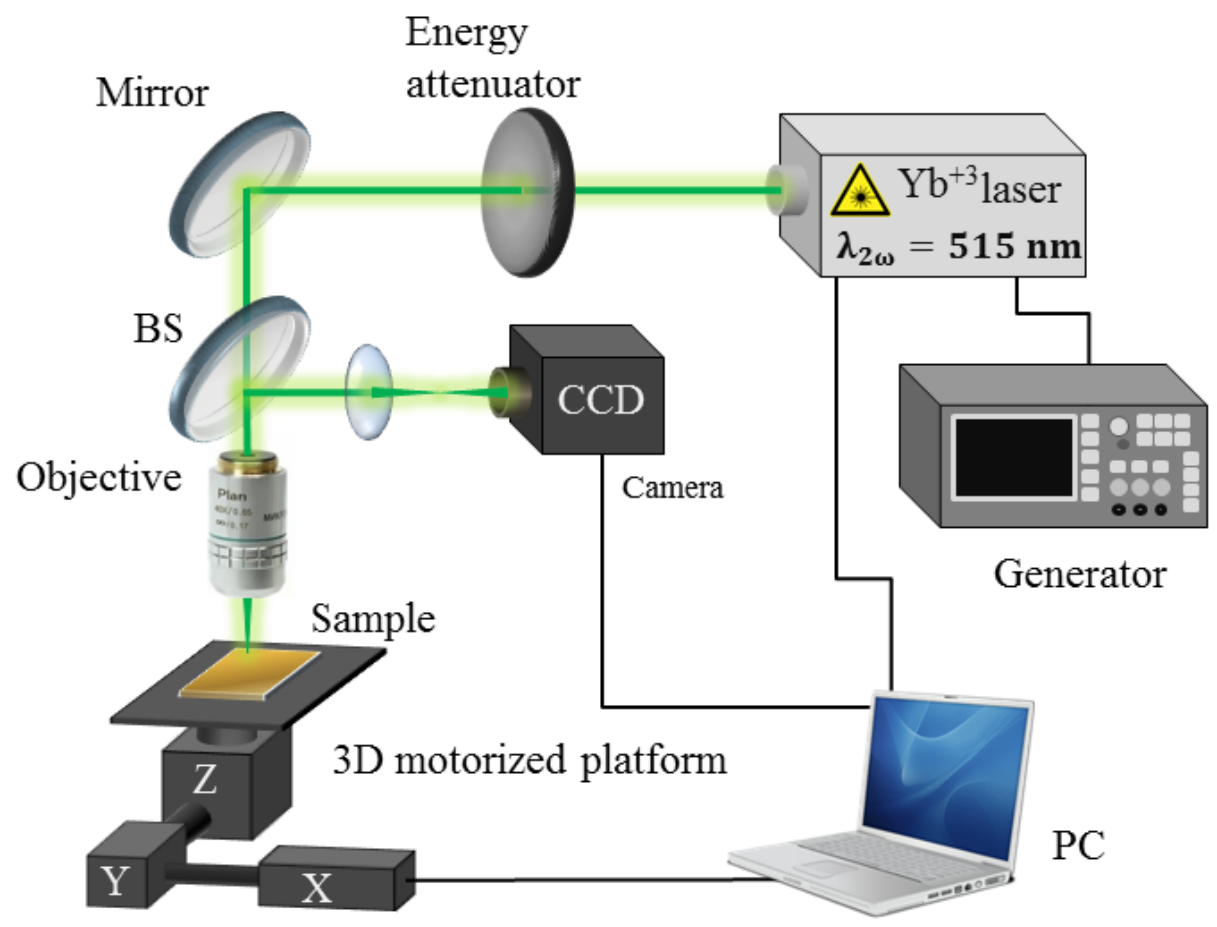

Fig. 1. Photoluminescence spectra of the blend bulk heterojunction materials films, excited at wavelength $345 \mathrm{~nm}$ (a) and wavelength $553 \mathrm{~nm}$ (b).

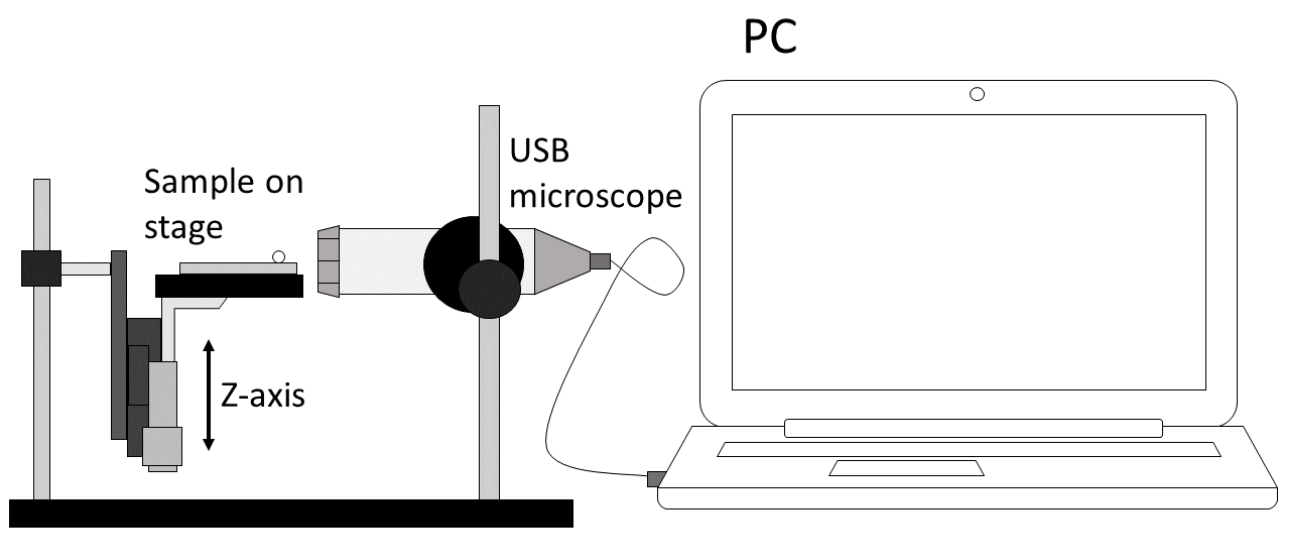

Fig. 2. Setup for the wetting angle measurements. 


\section{RESULTS AND DISCUSSION}

For our purpose, it was initially necessary to determine the optimal laser exposure regime (pulse energy and repetition rate, speed of 3D platform). In this regime, the top polymer layer should be detached from the bottom silver one, while the silver layer should remain on the substrate. Several lines at single pulse impact on the two layered film (Fig. 3) were made at different pulse energies on the sample surface at a pulse repetition rate of $1 \mathrm{kHz}$ and a platform movement speed of $12.5 \mu \mathrm{m}$ per second. Their SEM images show the craters on the surface of the polymer layer (large diameter) and the through holes on the silver layer (small diameter). These images also show how these diameters increase versus laser pulse energy (fluence). At certain energy, the holes on the silver film are no longer formed, and on the polymer film craters are still present. The plots showing dependence of a squared hole and craters radiuses against logarithm of laser pulse energy is given in Fig. 4. The angular slope $\mathrm{R}_{1 / e}$ of linear dependence $\mathrm{R}^{2}(\ln \mathrm{E})$ and the value of threshold energy $E_{a b l}$ are shown in Fig. 4. The ablation threshold for polymer layer of the film was $F_{a b l 2}=E_{a b l 2} / \pi R_{1 / e}^{2}=0.21 \mathrm{~J} / \mathrm{cm}^{2}$ and for both layers of the film (through hole formation) was $F_{a b l 1}=E_{a b l 1} / \pi R_{1 / e}^{2}=1.2 \mathrm{~J} / \mathrm{cm}^{2}$.

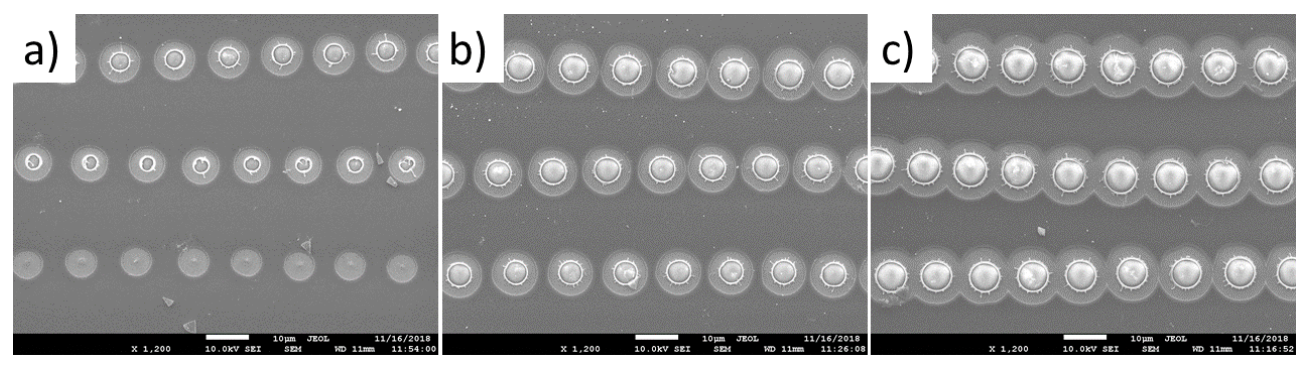

Fig. 3. SEM images of lines at single pulse impact on the two layered film at pulse energies equal to (bottom to top) a) 20, 40, $60 \mathrm{~nJ}$; b) 80, 100, $120 \mathrm{~nJ}$; c) 160, 240, $320 \mathrm{~nJ}$.
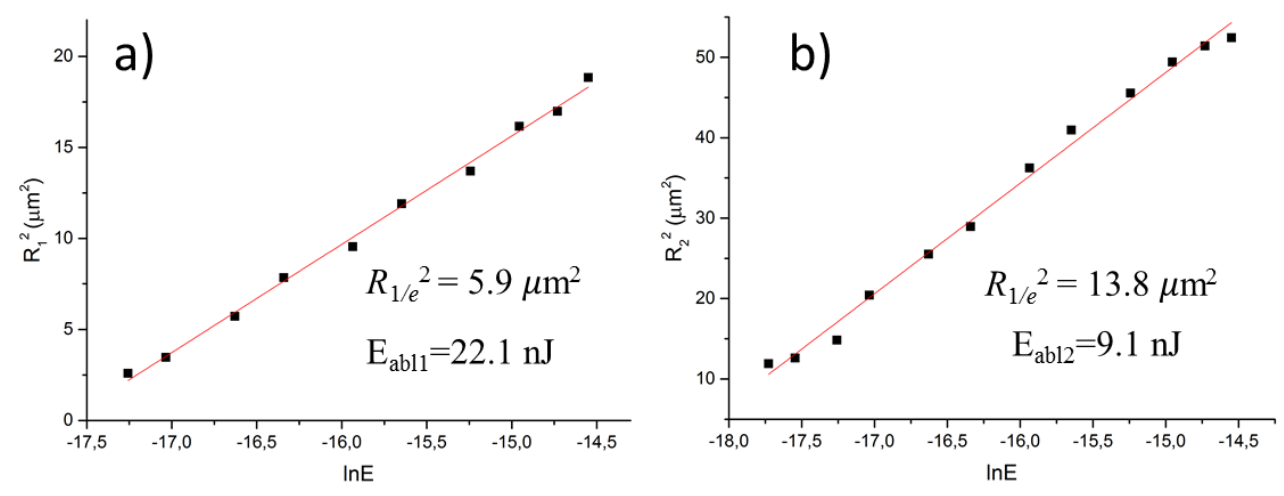

Fig. 4. Dependence of squared hole radius on natural logarithm of laser pulse energy for silver layer of the film (a); squared crater radius on natural logarithm of laser pulse energy for polymer layer of the film (b). 
After the choice of proper fs-laser micro-structuring regime, setting the laser pulse repetition rate and the speed of movement of the platform, the mode in which the structure is formed in the form of a continuous straight groove was chosen. Then, using an external generator that sets the pulse repetition rate of the laser depending on the frequency of movement of the platform, which is set programmatically with a computer, an array was produced in the form of periodic lines with a period of $\sim 7-8 \mu \mathrm{m}$. Such an array structure was observed with a scanning electron microscope (Fig.5). In this processing, the laser pulse energy of $18 \mathrm{~nJ}$, pulse repetition rate of 20 $\mathrm{kHz}$ and platform movement speed was $625 \mu \mathrm{m}$ per second are chosen. It is worth noting that the data period and the thickness of the structure could not be achieved by mechanical processing methods.
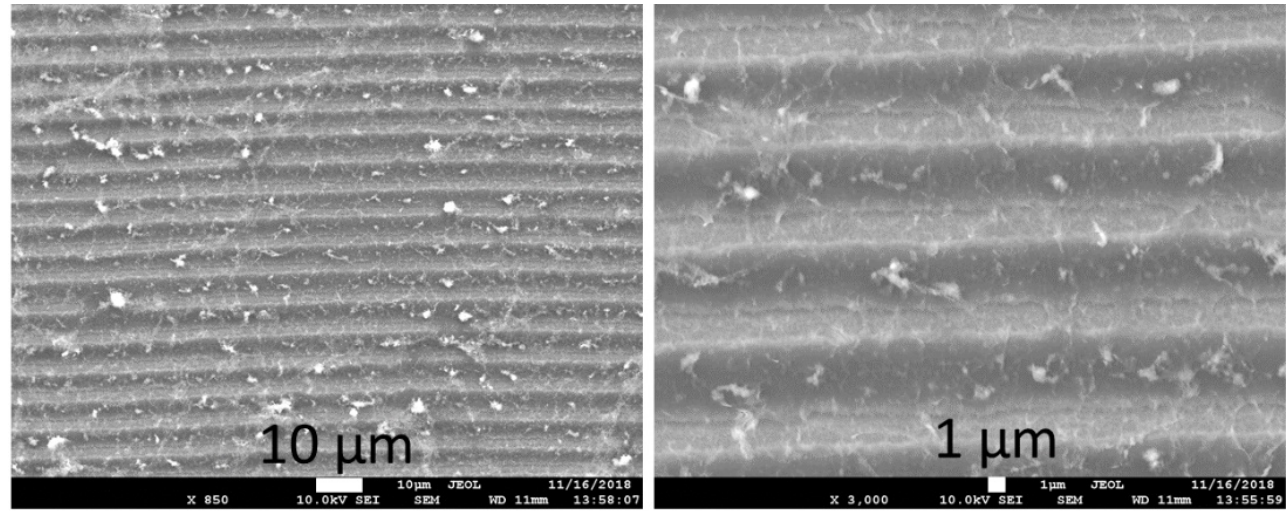

Fig. 5. Low magnification (left) and high magnification (right) SEM images of the structured film.
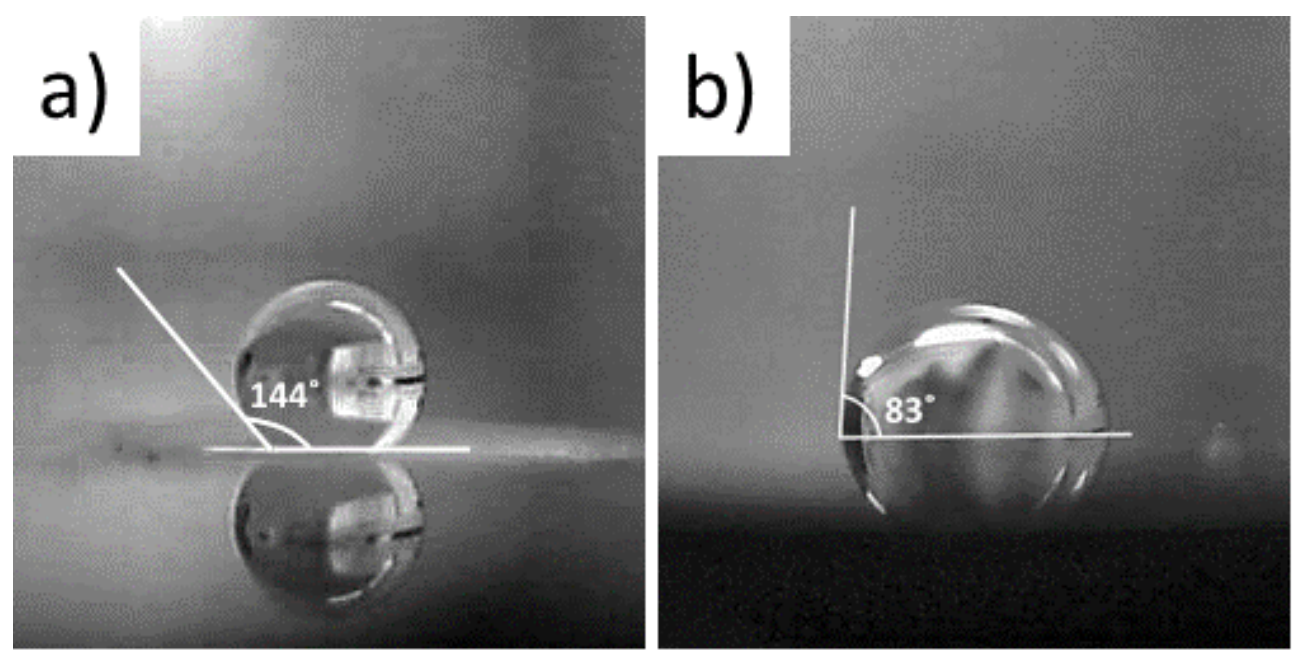

Fig. 6. Side-view images of deionized water micro-droplets of on a) the structured surface, b) the polymer surface. 
WETTING CONTROL IN THE LAYERED POLIMER-SILVER THIN FILM ...

After producing the array structure, a drop of de-ionized water with diameter of about 1.5 $\mathrm{mm}$, that much larger than the structure period, was placed on the micro-structured surface to measure the wetting angle. In Fig. 6a, it can be seen that the contact angle of the droplet with the surface was $144^{\circ}$. For comparison, a drop of deionized water was also placed on the surface of the polymer (Fig. 6b). The contact angle of the drop with the polymer surface was $83^{\circ}$. Thus, the micro-structuring of the film surface made it possible to change the wetting angle by $61^{\circ}$.

\section{CONCLUSION}

This paper presents the results of femtosecond laser microstructuring of a thin two-layered metal-polymer film, for directional wetting applications. The film micro-structured by the femtosecond laser pulses in the chosen regime exhibits the wetting angle of $144^{\circ}$ regarding droplets of deionized water, comparing to the wetting angle of $83^{\circ}$ for the raw polymer film. Hence, femtosecond laser micro-structuring of the two-layered metal-polymer film enables to govern its surface fluidic flows.

\section{REFERENCES}

[1] Jiale Yong, Qing Yang, Feng Chen, Dongshi Zhang, Umar Farooq, Guangqing Du and Xun Hou, J. Mater. Chem. A 2 (2014) 5499.

[2] Anne-Marie Kietzig, Mehr Negar Mirvakili, Saeid Kamal, Peter Englezos and Savvas G. Hatzikiriakos, Journal of Adhesion Science and Technology 25 (2011) 2789.

[3] D. H. Kam, S. Bhattacharya and J. Mazumder, J. Micromech. Microeng. 22 (2012) 105019.

[4] L. Boinovich, A. Domantovskiy, A. Emelyanenko, A. Pashinin, A. Ionin, S. Kudryashov and P. Saltuganov, ACS Applied Materials and Interfaces 6 (2014), 2080.

[5] D. Cristea, P. Obreja, M. Kusko, E. Manea and R. Rebigan, Materials Science and Engineering 26 (2006) 1049.

[6] D. A. Zayarnyi, A. A. Ionin, S. I. Kudryashov, S. V. Makarov, A. A. Rudenko, E. A. Drozdova, S. B. Odinokov, Quantum Electronics 45 (2015) 462.

[7] T. V. Baikova, P. A. Danilov, S. A. Gonchukov, V. M. Yermachenko, A. A. Ionin, R. A. Khmelnitskii, S. I. Kudryashov, T. T. H. Nguyen, A. A. Rudenko, I. N. Saraeva, T. S. Svistunova, and D. A. Zayarny, AIP Conference Proceedings 1874, 040052 (2017).

[8] D Pavlov, S Gurbatov, SI Kudryashov, PA Danilov, AP Porfirev, SN Khonina, OB Vitrik, SA Kulinich, M Lapine, AA Kuchmizhak, Optics Letters 44 (2019), 283.

[9] F. Gentile, G. Das, M. Coluccio, F. Mecarini, A. Accardo, L. Tirinato, R. Tallerico, G. Cojoc, C. Liberale, P. Candeloro et al., Microelectronic Engineering 87 (2010) 98.

[10] F. De Angelis, F. Gentile, F. Mecarini, G. Das, M. Moretti, P. Candeloro, M. Coluccio, G. Cojoc, A. Accardo, C. Liberale et al.,Nature Photonics 5 (2011) 682.

[11] Zhizhchenko, A., Kuchmizhak, A., Vitrik, O., Kulchin, Y., Juodkazis, S., Nanoscale 10 (2018) 21414. 